E. Paolini* and A. Tamagnini

\title{
Minimal cluster computation for four planar regions with the same area
}

https://doi.org/10.1515/geofl-2018-0007

Received September 15, 2017; accepted November 7, 2017

Abstract: The topology of a minimal cluster of four planar regions with equal areas and smallest possible perimeter was found in [9]. Here we describe the computation used to check that the symmetric cluster with the given topology is indeed the unique minimal cluster.

MSC: 49K10, 65K10, 51M25

\section{Introduction}

We consider the problem of enclosing and separating $N$ regions of $\mathbb{R}^{2}$ with prescribed area and with the minimal possible interface length.

The case $N=1$ corresponds to the celebrated isoperimetric problem whose solution, the circle, was known since antiquity.

For $N \geq 1$ first existence and partial regularity in $\mathbb{R}^{n}$ was given by Almgren [1] while Taylor [12] describes the singularities for minimizers in $\mathbb{R}^{3}$. Existence and regularity of minimizers in $\mathbb{R}^{2}$ was proved by Morgan [6] (see also [4]): the regions of a minimizer in $\mathbb{R}^{2}$ are delimited by a finite number of circular arcs which meet in triples at their end-points.

Foisy et al. [2] proved that for $N=2$ in $\mathbb{R}^{2}$ the two regions of any minimizer are delimited by three circular arcs joining in two points (standard double bubble) and are uniquely determined by their enclosed areas. Wichiramala [14] proved that for $N=3$ in $\mathbb{R}^{2}$ the three regions of any minimizer are delimited by six circular arcs joining in four points. Such configuration (standard triple bubble) is uniquely determined by the given enclosed areas, as shown by Montesinos [5].

Recently, in [9], the case of four regions with equal areas has been considered and in this case the topology of minimal cluster has been determined: the cluster is composed by four connected regions, two of them $E_{1}$, $E_{2}$ are quadrangular and are adjacent to each other, two of them $E_{3}, E_{4}$ are triangular and are adjacent to the two quadrangular regions. Such configuration is called sandwich and is depicted in Figure 1.

It is conjectured that in this topology there is a unique minimizer which is the cluster with two axes of symmetry: regions $E_{1}$ and $E_{2}$ are congruent to each other and the same is true for regions $E_{3}$ and $E_{4}$. In this paper we describe the computations backing such a conjecture. The actual code to produce the computation is available in [10].

*Corresponding Author: E. Paolini: Dipartimento di Matematica, Largo Bruno Pontecorvo 5, Università di Pisa, Italy E-mail: emanuele.paolini@unipi.it

A. Tamagnini: Dipartimento di Matematica e Informatica “U. Dini”, Viale Morgagni 67/a, Università degli Studi di Firenze, Italy, E-mail: andrea.tamagnini@unifi.it 


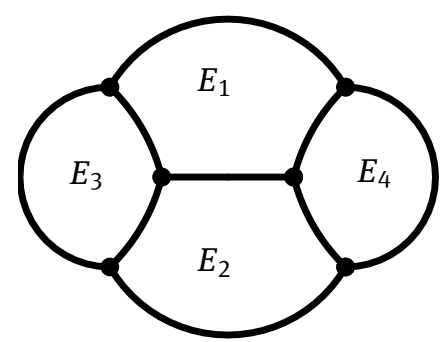

Figure 1: The symmetric cluster with sandwich topology.

\section{2 general results}

Let us denote with $\boldsymbol{E}=\left(E_{1}, \ldots, E_{N}\right)$ an $N$-uple of disjoint measurable subsets of $\mathbb{R}^{2}$. We will say that $\boldsymbol{E}$ is a cluster and that $E_{1}, \ldots, E_{N}$ are its regions. We define the external region $E_{0}$ as

$$
E_{0}=\mathbb{R}^{2} \backslash \bigcup_{i=1}^{N} E_{i} .
$$

The sets $E_{0}, E_{1}, \ldots, E_{N}$ are hence a partition of the whole plane $\mathbb{R}^{2}$.

We denote with $\boldsymbol{m}(\boldsymbol{E})=\left(\left|E_{1}\right|, \ldots,\left|E_{N}\right|\right)$ the $N$-uple of the measures of the regions of the cluster. Also we define the perimeter of the cluster as

$$
P(\boldsymbol{E}):=\frac{1}{2} \sum_{i=0}^{N} P\left(E_{i}\right)
$$

where $P\left(E_{i}\right)$ is the perimeter (the length of the boundary) of the measurable set $E_{i}$. The perimeter of the cluster represents the total length of the interfaces between the regions. Almost every point in the boundary of the cluster belongs to two adjacent regions, hence the factor $\frac{1}{2}$ in the previous definition.

We are interested in the problem of finding the clusters with minimal perimeter among all clusters with prescribed areas. Such clusters will be called minimal cluster.

The following result gives the existence of minimal clusters (see [1], [6] and [4]).

Theorem 2.1 (existence of minimal clusters). Given $\boldsymbol{a} \in \mathbb{R}_{+}^{N}$ there exists a cluster $\boldsymbol{E}$ in $\mathbb{R}^{n}$ such that $\boldsymbol{m}(\boldsymbol{E})=\boldsymbol{a}$ and such that

$$
P(\boldsymbol{E}) \leq P(\boldsymbol{F})
$$

for all $\boldsymbol{F}$ such that $\boldsymbol{m}(\boldsymbol{F})=\boldsymbol{a}$.

Minimal clusters have also very good regularity properties. In particular the structure of minimal cluster has been widely studied when the ambient space is $\mathbb{R}^{2}$ (see [6]) or $\mathbb{R}^{3}$ (see [12]). We recall the regularity result for the planar case.

Theorem 2.2 (regularity of planar clusters). Let $\boldsymbol{E}$ be a minimal cluster in $\mathbb{R}^{2}$. Then each region $E_{k}$ of $\boldsymbol{E}$ is composed by a finite number of connected components. Each connected component is delimited by a finite number of circular arcs or straight line segments. Each arc separates two components of different regions. The arcs meet in triples at their end points (which we call vertices) with equal angles of 120 degrees. The sum of the signed curvatures of the three arcs joining in a vertex is zero.

We will say that a cluster $\boldsymbol{E}$ satisfying the regularity properties stated in the previous theorem is stationary.

Of course stationarity is preserved under isometries and rescalings of the plane. One can check (see [13], [5], [15], [11]) that stationarity is also preserved by circle inversion: if three circular arcs meet in a point with equal angles of 120 degrees of course their circular inversion are also arcs (or straight line segments) which also meet in a point with the angles preserved. But not only that: if the sum of the signed curvatures is zero, 
this condition is also preserved under circular inversion (while the single curvatures might change). This means that stationarity of clusters is preserved by all Moebius transformations of the plane. So, if a stationary cluster is obtained as the stereographic projection of a partition on the sphere, we can rotate the sphere as we like, project back the partition to the plane and obtain another stationary cluster.

In particular it happens (see [5]) that all double bubbles (i.e. the minimal clusters with two regions, see [2]) can be obtained, up to rescaling, by projecting the geodesic network on the sphere given by three meridians joining in two antipodal points with equal angles of 120 degrees. All triple bubbles (i.e. the minimal clusters with three regions, see [15]) are obtained, up to rescaling, by projecting the geodesic network of a regular tetrahedron on the sphere.

To further investigate the geometry of minimal clusters we point out a general result which can be stated for a triangular region of any stationary cluster (see [15]).

Theorem 2.3 (removal of triangular regions). Let $T$ be a triangular region of a stationary cluster $\boldsymbol{E}$. Consider the three arcs which meet the three vertices of $T$ and are not edges of $T$. The sum of the signed curvatures of these three arcs is zero. Moreover, if prolonged inside $T$, these arcs meet in a point $P$ inside $T$ with equal angles of 120 degrees. Hence, if we remove the triangle $T$ and prolong the three arcs, we obtain a new stationary cluster.

To explicitly compute the area of a region bounded by circular arcs we develop some formulas. We are going to use complex numbers to represent the points of $\mathbb{R}^{2}$.

Let $z$ and $w$ be any two points on the complex plane and consider the circular arc going from $z$ to $w$ which has direction $\beta$ in the point $z$ (i.e. the half line starting from $z$ tangent to the arc towards $w$ identifies and angle $\beta$ with respect to the axis of positive real numbers).

We are going to separately compute the area of the trapezoid below the straight segment $z w$ and the area of the circle segment bounded by the arc and the line segment. We need to compute the oriented area, so we consider the area positive if the path going from $z$ to $w$ along the arc and then following the trapezoid is counter-clockwise oriented.

To compute the area of the circle segment we need to find the angle $\alpha$ between the arc and the chord going from $z$ to $w$. The angle of the $\operatorname{arc}$ is $\theta$ and the angle of the chord is ${ }^{1} \arg (w-z)$ hence

$$
\alpha=\theta-\arg (w-z) .
$$

Since $\arg (w-z)$ is defined up to multiples of $2 \pi$ we require that $\alpha \in[-\pi, \pi]$ by possibly adding an appropriate multiple of $2 \pi$. Notice that the arc spans an angle $2 \alpha$ on the circle containing it. So we can compute the radius of the circle

$$
R=\frac{|w-z|}{2 \cdot \sin (\alpha)}
$$

and the area of the circular sector is $\alpha R^{2}$ while the area of the circular segment is obtained removing the area of the triangle with angle $2 \alpha$ between two equal sides of length $R$ :

$$
\alpha R^{2}-R^{2} \sin \alpha \cos \alpha=R^{2}(\alpha-\sin \alpha \cos \alpha) .
$$

Notice that when $\alpha$ is negative the area computed above is also negative. This is correct because the region below the arc (imagine that $z$ is on the right with respect to $w$ ) is concave and should hence be removed from the area of the trapezoid.

The area of the trapezoid below the straight segment $z w$ can be easily computed as

$$
\frac{1}{2} \operatorname{Re}(z-w) \operatorname{Im}(z+w)=\frac{1}{8}\left(z^{2}-\bar{z}^{2}\right)\left(w^{2}-\bar{w}^{2}\right) .
$$

We have hence found the signed area below the $\operatorname{arc} z w$ as

$$
A(z, w, \theta)=\frac{1}{8}\left(z^{2}-\bar{z}^{2}\right)\left(w^{2}-\bar{w}^{2}\right)+R^{2}(\alpha-\sin \alpha \cos \alpha)
$$

1 Remember $\arg z$ is such an angle that $z=|z| \cdot \exp (i \cdot \arg (z))$. 
where $R$ and $\alpha$ are obtained by $z, w$ and $\theta$ as described above.

Another computation we will need in the following, is a formula to find the direction of an arc after a circular inversion is performed.

In general suppose to have an $\operatorname{arc} \Gamma$ starting at point $z$ in direction $\alpha$. The angle $\alpha$ is the angle between $\Gamma$ and the ray $H$ starting in $z$ and with the same direction as the positive real numbers (horizontal ray from $z$ ). Let $z^{\prime}=1 / \bar{z}$ be the circular inversion of the point $z$. Now consider the ray $R$ starting from 0 and passing trought $z$. This ray has direction $\beta=\arg z$. The angle $\alpha$ between $\Gamma$ and $H$ is the sum $\alpha=\gamma+\beta$ where $\gamma$ is the (oriented) angle between $R$ and $\Gamma$. We want to compute the angle $\alpha^{\prime}$ in $z^{\prime}$ between the inversion $\Gamma^{\prime}$ of $\Gamma$ and the inversion $H^{\prime}$ of $H$. We know that $R^{\prime}=R$ but the direction of $R^{\prime}$ in $z^{\prime}$ is opposite to the direction of $R$ in $z$ : it is $\pi+\beta$. From this direction we subtract $\gamma$ to find the direction $\alpha^{\prime}$ of $\Gamma^{\prime}$. But since $\gamma=\alpha-\beta$ we finally obtain

$$
\alpha^{\prime}=\pi+\beta-\gamma=\pi+2 \beta-\alpha=\pi+2 \arg (z)-\alpha .
$$

\section{Minimal planar clusters with four regions of equal area}

From now on we will consider the particular case of four planar regions with equal area. In the recent paper [9] we have obtained the following result.

Theorem 3.1. Let $\boldsymbol{E}=\left(E_{1}, E_{2}, E_{3}, E_{4}\right)$ be a cluster with $N=4$ regions in the plane which is minimal with prescribed equal areas $\boldsymbol{a}=(a, a, a, a)$. Then all the four regions are connected. Moreover two of them (say $E_{1}$ and $E_{2}$ ) have four edges and two of them (say $E_{3}$ and $E_{4}$ ) have three edges.

Consider now the minimal cluster $\boldsymbol{E}$ with four planar regions $E_{1}, E_{2}, E_{3}$ and $E_{4}$ of equal area. Suppose $E_{1}$ and $E_{2}$ are the quadrangular regions while $E_{3}$ and $E_{4}$ are triangular. If we remove both triangular regions $E_{3}$ and $E_{4}$ prolonging the three remaining arcs we obtain a double bubble delimited by three arcs and two vertices.

Let us identify the ambient plane with the set of complex numbers $\mathbb{C}$. Up to translating, rotating and rescaling we might suppose that the two vertices of the resulting double bubble are the two complex numbers 0 and 1 . Suppose also that $E_{1}$ is the upper quadrangular region, $E_{2}$ is the lower quadrangular, $E_{3}$ is the right hand side triangular and $E_{4}$ is the left hand side triangular region (see Figure 2).

If we now perform the circular inversion $z \mapsto 1 / \bar{z}$ the vertex in 1 remains fixed, the vertex in 0 goes to $\infty$ and the three arcs joining the two vertices become three straight half lines going from 1 to $\infty$ with equal angles of 120 degrees. Let us call $\theta$ the direction (angle with respect to the positive real axis) of the half line which is obtained as the inversion of the arc which is the interface between the two regions of the double bubble. The triangular component $E_{3}$ around the vertex at 1 becomes a Releaux triangle centered in 1 i.e. a region bounded by three isometric circular arcs with vertices on the straight lines, such that the arc between two vertices has center on the third vertex. The Releaux triangle can be uniquely determined by means of the distance of its vertices from the center: let call $\rho$ such a distance.

We will now show that the whole minimal cluster $\boldsymbol{E}$ is determined by the two parameters $\theta$ and $\rho$. Let us explicitly compute the coordinates of the vertices of $\boldsymbol{E}$.

We start by identifying the vertices of the Releaux triangle, they are:

$$
z_{k}=1+\rho \cdot \exp \left(i \theta+\frac{2}{3} i \pi k\right), \quad k=0,1,2 .
$$

The corresponding vertices of $E_{3}$ are the circle inversion of the vertices of the Releaux triangle:

$$
w_{k}=\frac{1}{\bar{z}_{k}} \text {. }
$$

More precisely $w_{0}$ is the right hand side internal vertex of the cluster, and then $w_{1}$ and $w_{2}$ are the other two vertices of the triangular component in clockwise order.

Notice that the Releaux triangle with vertices $z_{0}, z_{1}, z_{2}$ is increasing (in the sense of set inclusion) with respect to the parameter $\rho$. Hence also the triangular region $E_{3}$ (which is the inversion of the Releaux triangle) 


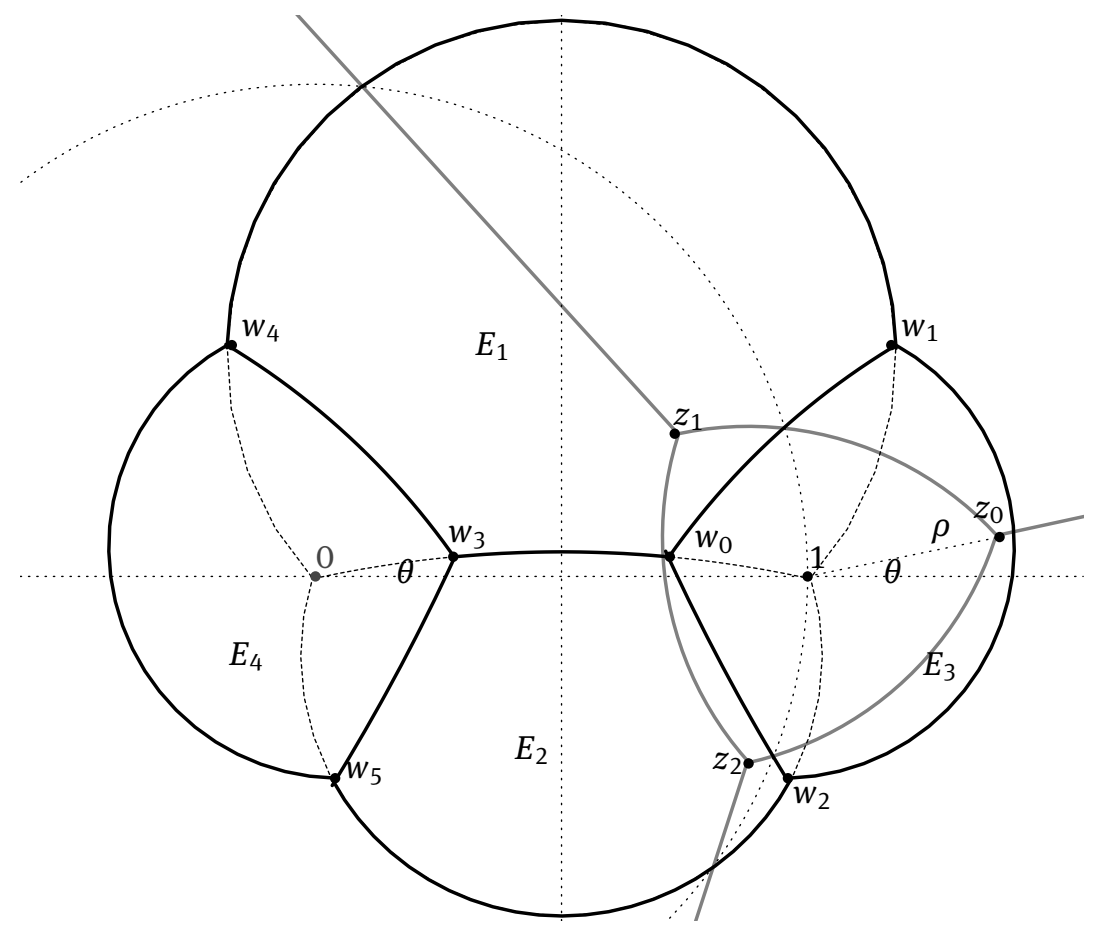

Figure 2: Cluster construction by means of circular inversion.

is increasing in $\rho$. This means that the area of such region is also increasing in $\rho$. So, if the area of the region is fixed, then $\rho$ is uniquely determined. As a consequence the two regions $E_{1}, E_{2}$ and their areas $\left|E_{1}\right|,\left|E_{2}\right|$ are decreasing in $\rho$.

Also, if we repeat the construction with the triangle centered in 0 , if we impose that the two triangles have the same area we find that they correspond to the same parameter $\rho$ because the double bubble is symmetric with respect to the axis $x=1 / 2$. This means that the minimal cluster $\boldsymbol{E}$ is itself symmetric with respect to the axis $x=1 / 2$ and the left hand side triangular region $E_{4}$ can be obtained by symmetry once $E_{3}$ has been determined. More precisely the three vertices of $E_{4}$ are given by

$$
w_{k+3}=\sigma\left(w_{k}\right), \quad k=0,1,2
$$

where $\sigma$ is the symmetry $\sigma(x+i y)=(1-x)+i y$. The point $w_{3}$ is the internal vertex of $\boldsymbol{E}$ on the left hand side and $w_{4}, w_{5}$ are the other two vertices of $E_{4}$ in counter-clockwise order.

All the coordinates of the vertices of $\boldsymbol{E}$ have been found as a function of the two parameters $\theta$ and $\rho$. We are now going to compute the areas of the regions. To compute the areas of the regions of the cluster $\boldsymbol{E}$ using formula (1) we now need to find the direction of the arcs bounding each region. We know which are the directions of the arcs bounding the Releaux triangle, so we are going to use the formula (2) to compute the direction of the circular inversion of an arc.

This formula allows us to compute the direction of the three arcs which start from the three vertices $w_{0}, w_{1}, w_{2}$ and end on the three vertices of the left hand side triangular region $w_{3}, w_{4}, w_{5}$. These directions are

$$
\beta_{k}=\pi+2 \arg \left(z_{k}\right)-\left(\theta+\frac{2}{3} k \pi\right), \quad k=0,1,2 .
$$

The direction of the arcs bounding the triangular region can be computed accordingly, since the three arcs joining in a vertex form equal angles of 120 degrees.

So, the area of the right hand side triangular region $E_{3}$ (which is equal to the area of the other triangular region $\left.E_{4}\right)$ is given as a function of $\theta$ and $\rho$ :

$$
\left|E_{3}\right|=\left|E_{4}\right|=A\left(w_{0}, w_{1}, \beta_{0}+2 \pi / 3\right)+A\left(w_{1}, w_{2}, \beta_{1}+2 \pi / 3\right)+A\left(w_{2}, w_{0}, \beta_{2}+2 \pi / 3\right) .
$$


The area of the upper region $E_{1}$ is given by

$$
\left|E_{1}\right|=2 A\left(w_{0}, w_{2}, \beta_{0}-2 \pi / 3\right) A\left(w_{2}, w_{5}, \beta_{1}\right)-A\left(w_{0}, w_{3}, \beta_{0}\right)
$$

and the area of the lower region $E_{2}$ is given by

$$
\left|E_{2}\right|=-2 A\left(w_{0}, w_{1}, \beta_{0}+2 \pi / 3\right)-A\left(w_{1}, w_{4}, \beta_{1}\right)+A\left(w_{0}, w_{3}, \beta_{0}\right)
$$

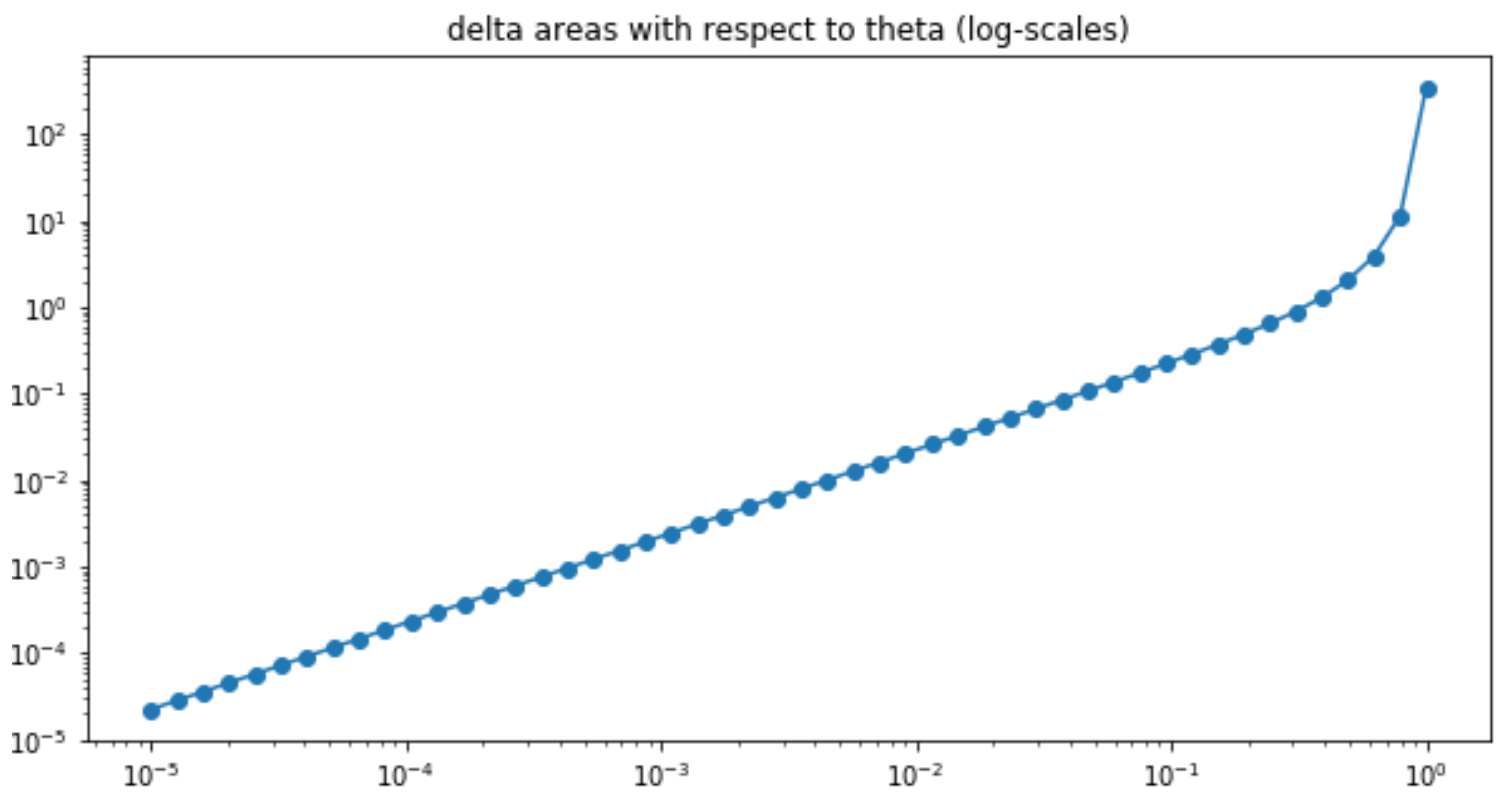

Figure 3: Plot of $\left|E_{1}\right|-\left|E_{2}\right|$ with respect to parameter $\theta$.

The formulas for $\left|E_{1}\right|,\left|E_{2}\right|$ and $\left|E_{3}\right|=\left|E_{4}\right|$ are ultimately functions of $\theta$ and $\rho$. Recall also that for every $\theta$ the area $\left|E_{3}\right|$ is increasing in $\rho$ while $\left|E_{1}\right|$ and $\left|E_{2}\right|$ are decreasing in $\rho$. This allows us to find $\rho=\rho(\theta)$ such that $\left|E_{3}\right|=\left|E_{2}\right|$ using a simple bisection method since $\left|E_{3}\right|-\left|E_{2}\right|$ is decreasing in $\rho$. Fixing $\rho=\rho(\theta)$ we have now guaranteed that $\left|E_{2}\right|=\left|E_{3}\right|=\left|E_{4}\right|$ and we only need to find $\theta$ such that $\left|E_{1}\right|=\left|E_{2}\right|$. Notice that for $\theta=0$ the cluster is (obviously) symmetric with respect to the real axis so we already know that in this case all the regions have equal areas.

What is missing is a proof that $\left|E_{1}\right|-\left|E_{2}\right|$ is increasing in $\theta$. This would prove that $\theta=0$ is the only possible solution and hence that the cluster $\boldsymbol{E}$ is uniquely determined and is symmetric also with respect to the real axis (we already know that it is symmetric with respect to $x=1 / 2$ ).

With the computations gathered so far we are able to numerically compute the function $\left|E_{1}\right|-\left|E_{0}\right|$ as a function of $\theta$ and have a numerical proof that such a function is increasing as shown in Figure 3.

\section{References}

[1] F. J. Almgren: Existence and regularity almost everywhere of solutions to elliptic variation problems viwh constraints, Mem. AMS 165 (1976).

[2] J. Foisy, M. Alfaro, J. Brock, N. Hodges, J. Zimba: The standard soap bubble in $\mathbb{R}^{2}$ uniquely minimizes perimeter, Pacific J.Math 159, 47-59 (1993).

[3] M. Hutchings, F. Morgan, M. Ritoré, A. Ros: Proof of the double bubble conjecture, Annals of Math, 155(2), 459-489 (2002). 
[4] F. Maggi: Sets of finite perimeter and geometric variational problems, Volume 135 of Cambridge Studies in Advanced Mathematics, Cambridge University Press, Cambridge (2012).

[5] A. Montesinos Amilibia: Existence and uniqueness of standard bubble clusters of given volumes in $\mathbb{R}^{N}$, Asian J. Math 5(1), 25-32 (2001).

[6] F. Morgan: Soap bubbles in $\mathbb{R}^{2}$ and in surfaces, Pacific J.Math 165, 347-361 (1994).

[7] F. Morgan, J. M. Sullivan: Open problems in soap bubble geometry, Int. J.Math 7, 883-842 (1996).

[8] F. Morgan, W. Wichiramala: The standard double bubble is the unique stable double bubble in $\mathbb{R}^{2}$, Proc. Amer. Math. Soc., 130(9), 2745-2751 (2002).

[9] E. Paolini, A. Tamagnini: Minimal clusters of four planar regions with the same area, ESAIM: COCV, online first https://doi.org/10.1051/cocv/2017066.

[10] E. Paolini, A. Tamagnini: Sandwich cluster analysis, GitHub repository, https://github.com/paolini/sandwich/ (2017).

[11] A. Tamagnini: Planar Clusters, Ph.D. thesis, University of Florence, http://cvgmt. sns . it/paper/2967/ (2016).

[12] J. E. Taylor: The structure of singularities in soap-bubble-like and soapfilm- like minimal surfaces, Ann. Math., 103, 489-539 (1976).

[13] R. Vaughn: Planar soap bubbles, Ph.D. thesis, University of California, Davis (1998).

[14] W. Wichiramala: The Planar triple bubble problem, Ph.D. thesis, University of Illinois, Urbana-Champ (2002).

[15] W. Wichiramala: Proof of the planar triple bubble conjecture, J. reine angew. Math. 567, 1-49 (2004). 